\title{
A brief history of surgery for peripheral nerve sheath tumors
}

\author{
Ciaran J. Powers, M.D., Ph.D., And Allan H. Friedman, M.D. \\ Division of Neurosurgery, Department of Surgery, Duke University Medical Center, Durham, \\ North Carolina
}

\begin{abstract}
$\checkmark$ The authors present a brief and selective history of surgery for peripheral nerve tumors to illustrate how the current understanding of the nature of disease influences the choice of surgical intervention. There was very little understanding of the anatomy and function of peripheral nerves in ancient times; consequently, surgical treatments for peripheral nerve tumors were based on the writings of authorities. The confusion between traumatic neuromas and genuine nerve sheath tumors coupled with the belief that manipulation of a peripheral nerve might be lethal to the patient stifled the development of surgical techniques for the management of nerve tumors in the 18th and 19th centuries. It was not until the 20th century, with an increased understanding of the microscopic anatomy of nerve sheath tumors, that efficacious surgical treatments for these diseases were developed. Continued advances in the understanding of the biology of these tumors will continue to impact their surgical management.
\end{abstract}

KEY WORDS • history • peripheral nerve sheath tumor • surgery

$\mathrm{T}$ HE rational application of any surgical intervention for a particular disease is guided by the understanding of the nature of that disease. This is clearly demonstrated in a review of the history of surgery for PNSTs. The Ancients believed atraumatic illnesses to be due to the will of the gods, and as such there was no real role for surgical intervention. The belief that manipulating nerves could be lethal also hampered the development of surgical techniques for peripheral nerve tumors. The surgical management of PNSTs in the 17th and 19th centuries became more popular with a better understanding of the basic anatomy and distinction between traumatic neuromas and primary tumors. Finally, management of PNSTs in the 20th century is guided by our understanding of the distinctions among schwannomas, neurofibromas, and other tumors that affect the peripheral nerves. In this brief review, we highlight how the surgical management of PNSTs has been guided by changes in the understanding of the nature of these tumors.

\section{Ancient History: Tendons and Nerves}

It is difficult to interpret the writings from antiquity concerning peripheral nerve tumors, and often impossible to correlate the descriptions of diseases in general in a modern medical textbook with those mentioned in ancient writings. ${ }^{23}$ Furthermore, the descriptions of peripheral nerve le-

Abbreviations used in this paper: $\mathrm{NF} 1=$ neurofibromatosis Type $1 ;$ PNST = peripheral nerve sheath tumor. sions in ancient writings are predominantly about traumatic injuries; peripheral nerve tumors were simply too rare to have generated a significant amount of literature. Consequently, a review of the ancient literature reveals only vague references to peripheral nerve tumors..$^{39}$ The ancient Greeks did not usually perform dissections of the human body, and their understanding of anatomy was quite rudimentary. ${ }^{27}$ There seems to have been confusion regarding the structural and functional differences between nerves and tendons. In translations of Homer's Iliad, the word " $\tau \in \nu 0 \nu \tau \epsilon$ " has been interpreted to mean either "tendon" or "nerve," and is translated accordingly based on its context. ${ }^{27}$ Furthermore, because spontaneous illnesses were thought to be the will of the gods, management was directed more towards the divine rather than some physical intervention. ${ }^{19}$ This is in contradistinction to the ancient management of traumatic injury, the cause of which was obvious and the treatment more pragmatic. ${ }^{27}$

The Hippocratic writings (circa 460-375 BC) reveal a slightly more sophisticated understanding of nerves themselves, although there was still confusion over the difference between tendons and nerves. In his commentary on translating the Hippocratic writings, The Nature of the Child and The Heart, I. M. Lonie describes the ambiguity of words used to describe nerves and tendons. ${ }^{17}$ Based on the book of Aphorisms, it is clear that the Hippocratic authors were aware that nerves do not spontaneously regenerate after resection. In Section VI of Aphorisms, the authors state that "division of bone, cartilage, nerve, the delicate 
part of the jaw, or of the foreskin is not followed by growing and joining together again." This concept is repeated in Section VII, with the statement that "bones, cartilages and nerves, when divided, will not reconstitute themselves."17 The Hippocratic writings also contain the idea that "cold is bad for nerves, heat is good," a concept put forth in Aphorisms Section V. ${ }^{17}$ This provided the basis for a medical management of nerve injury: if the cause of nerve dysfunction was cold, heat might improve nerve function.

The ancient Greek author Herophilus (circa 335-280 BC) contributed to a greater understanding of peripheral nerves and their functions through dissections of human cadavers. He distinguished nerves from tendons and traced their origin back to the spinal cord. ${ }^{7}$ Unfortunately, his writings were lost in the fire of the library at Alexandria in 272 AD.

Galen of Pergamon (129-200 AD) based his understanding of peripheral nerves and their function on anatomical dissections, often of pigs and apes. He wrote detailed descriptions of the anatomy of peripheral nerves from the spinal cord out to the small digital nerves. Galen believed that once they were divided, peripheral nerves would not regenerate, ${ }^{16}$ and held that a wounded nerve in continuity with the brain would cause convulsions. Galen recommended an unction of ground earthworms to treat nerve injury rather than surgical repair. ${ }^{39}$

The ninth century Islamic physician Rhazes wrote extensively on neuroanatomy and was one of the first writers to assign separate motor and sensory functions to nerves. In Kitab al-hawi Fi Al-Tibb, ${ }^{31}$ Rhazes described how crosssectioning a nerve leads to a loss of function while longitudinal sectioning does not:

You have to be knowledgeable of the nerves that serve each organ. Some of the nerves are nerves for sensation; some of other nerves have motor function. The nerve that innervates the skin has a sensory function whereas the nerve that goes to tendon (muscle) has a motor function. The nerve function is abolished when the nerve is totally sectioned from a contusion, or compression, edema or tumor or from a severe cold that affects it. However, nerve lesions from edema, compression or cold may be reversed when the cause is treated. If there is a section in the body of the nerve as its middle part (cross section), paralysis of organs in that area occurs; if the nerve has longitudinal section (no loss of continuity of the nerve) organs are not damaged at all. Always go, in case of loss of sensation or movement by an organ, to the origin of the corresponding nerve, if it is cold warm it, if it is edema put some medication, if there is section of the nerve (total loss of nerve continuity) there is no therapy.

Rhazes echoed the Hippocratic idea of using heat to improve nerve dysfunction caused by cold. However, in cases in which the nerve was completely divided, Rhazes had a nihilistic perspective on treatment, and did not introduce any surgical options. Thus, although there was gradual advancement in the scientific understanding of peripheral nerves in ancient times, this progress did not lead to the rational surgical management of nerve injury or disease.

\section{Eighteenth and Nineteenth Centuries: Traumatic Neuromas and Nerve Tumors}

The idea that nerves could regenerate was not established until the end of the 18th century. ${ }^{2}$ Working in Hunter's laboratory in 1776, Cruickshank severed the vagal nerve in a dog; 2 weeks later he severed the contralateral vagal nerve, and the dog died. When he examined the site where the first vagal nerve had been severed, he noted a white substance which he did not think was scar tissue, but possibly regenerating nerve tissue. When he increased the time interval between severing the first and the contralateral vagal nerve, the animals survived. His work was presented to the Royal Society as evidence that nerves could regenerate but because his thesis rejected 1700 years of belief, it was rejected for publication. Nineteen years later, Haighton ${ }^{9}$ repeated these experiments. He cut one vagal nerve and some weeks later cut the second. After the dog was shown to have survived, Haighton went back and recut the first vagal nerve; the dog subsequently died. After this series of experiments, both Cruickshank and Haighton's manuscripts were published in 1795.

Most reports of nerve tumors in the 17th century concerned the swellings found on the ends of transected nerves, as is described by Lower in $1669 .{ }^{39}$ Odier of Geneva ${ }^{24}$ first applied the term "neuroma" to tumors formed by diseased enlargements of nerves. One of the first true descriptions of the surgical management of a PNST can probably be credited to Cheselden in 1741. In The Anatomy of the Human Body, Cheselden ${ }^{1}$ described a tumor that occupied the center of a "cubital" nerve, displacing the nerve fibers to the periphery. This seems to be a description of a schwannoma, but there is not enough clinical or histological information to draw this conclusion definitively. Despite recognizing the distinction between the nerve fibers and the tumor tissue itself, Cheselden seems to have excised the tumor en bloc with the nerve. In his extensive review of peripheral nerve tumors, Woods noted that "a number of nervous fasciculi, sometimes a good deal flattened can be traced, in a perfect state of continuity, running over its surface, to be connected below with the trunk of the nerve." As is typical of any major scientific advancement, new ideas that contradicted the dogma of the time were overlooked, only to be rediscovered later. Home recognized that some nerve tumors might be dissected free from the nerve fibers. In 1800 Home $^{12}$ described witnessing Hunter perform an en bloc resection of a tumor of the musculocutaneous nerve. Home examined the specimen in the laboratory and realized that the tumor could easily have been removed while keeping the parent nerve intact, concluding that en bloc resection of nerve tumors was unnecessary. Home subsequently put this theory to the test when he encountered a patient with an axillary nerve tumor. In his article he described incising the tumor and enucleating it with finger dissection. Unfortunately, the patient died on the postoperative Day 7, and at autopsy no cause of death was revealed other than inflammation at the operative site. Subsequently, it was considered too dangerous to dissect a tumor from a peripheral nerve, and both Swan ${ }^{34}$ in 1820 and Wood ${ }^{40}$ in 1829 discouraged the practice. Wood speculates that

...the death may perhaps be ascribed more to the general constitutional attention caused by the inflammation excited by the important parts seated in the axillary than from anything peculiar in the nervous connection of the tumor. It is worth consideration, at least, how far the result might have been different, if it had been possible to have removed the tumor along with the cyst, by the more simple operation of dividing the affected nerve in two places.

Wood echoed the practice of his time of treating periph- 
eral nerve tumors by "extirpation of the diseased part alone, and when essentially necessary to have recourse to removal of the affected limb." 40

As there was confusion in distinguishing between traumatic neuromas and nerve tumors, the approach of en bloc resection was applied to the management of both. In addition, many authors believed that inflammation of a peripheral nerve could be fatal to the patient. ${ }^{39}$ In 1813, Denmark ${ }^{4}$ treated a patient with a painful neuroma of the radial nerve from a musket ball wound with amputation of the arm. It was not until he examined the specimen and found a piece of the bullet in the neuroma that he questioned if the procedure had been too radical. Commentators on this case appear to have concluded that Mr. Denmark's case was simply a typical nerve tumor that resulted from trauma. ${ }^{39}$

As late as 1855 , James Syme ${ }^{35}$ reported treating peripheral nerve tumors with amputation. In The Lancet, Syme described three patients with peripheral nerve tumors and their management with amputation of the affected limb. Syme's strategy for managing these tumors is articulated clearly in his description of his first patient, a 43-year-old farm laborer who had "a swelling of the forearm, on the palmar side, a little above the wrist, about as large as a hen's egg." It is unclear if this was a traumatic neuroma or a genuine nerve tumor, as the patient had suffered blunt trauma to the area several years previously. Syme assessed the patient as having a neuroma of the median nerve and wrote: "I think before resorting to removal of the hand, an attempt should be made to dissect out the tumor; but the chance of success in accomplishing this being very small, the patient has given his consent to amputation if it should prove necessary." Unfortunately, at the time of operation Syme found the tumor "too intimately connected with the surrounding parts to admit of removal without amputation" 38 and he amputated the patient's hand at the midforearm. On examining the specimen, he found "the tumor itself was of roundish form, of yellow-white color, and pretty firm consistency. The fibers of the nerve were beautifully expanded over its surface."

Although Odier first used the term "neurom" in $1811^{24}$ to describe deep-seated, circumscribed nerve tumors, many other authors applied it indiscriminately to both primary and metastatic tumors involving nerves, as well as to traumatic neuromas. ${ }^{39}$ Between 1829 and 1842 , Cruveilhier $^{3}$ used the same term in his illustrations of peripheral nerve tumors and the swollen ends of nerves in amputation stumps. In 1849 R. W. Smith ${ }^{30}$ articulated the differences between traumatic, solitary, and multiple neuromas. Nevertheless, Smith continued to advocate en bloc resection of nerve tumors over enucleation for fear of causing inflammation of the nerve and death of the patient. Arguably the most meaningful insight into the management of nerve tumors was made by Virchow ${ }^{37}$ in 1864 when he divided tumors of the peripheral nerves into "true" and "false" neuromas. "True" neuromas contained both nerve fibers and elements of the nerve sheath, while "false" neuromas contained only the elements of the nerve sheath. This allowed clinicians to distinguish between traumatic neuromas and true peripheral nerve sheath tumors. ${ }^{39}$

In descriptions of the management of nerve tumors, some authors began to apply the increasingly sophisticated understanding of nerve response to traumatic injury and the role of reestablishing continuity to a transected nerve. As noted by Walker ${ }^{39}$, Michion in 1849 and Nelaton in 1863 described the management of tumors of the sciatic and median nerves, respectively, with en bloc resection followed by reapproximation of the nerve ends. Nelaton reported removing a neuroma of the median nerve and reapproximating the cut nerve ends with two silver wires. He reported that on Day 7 postoperatively the patient could move all of her fingers and oppose her thumb. This report led surgeons to believe that the median nerve regained function in only 7 days. ${ }^{14}$ Amputation as the primary treatment for PNSTs became increasingly less popular, and in $1887 \mathrm{Krause}^{15}$ advocated simple excision for well-encapsulated nerve tumors, reserving amputation for recurrent nerve tumors.

\section{Twentieth Century: Schwannomas and Neurofibromas}

Debate over the cell of origin of PNSTs began in the late 19th century. In 1869 , Genersich ${ }^{6}$ first proposed that "false" neuromas (using Virchow's nomenclature) arose from Schwann cells. Von Recklinghausen ${ }^{38}$ hypothesized in 1882 that peripheral nerve sheath tumors were derived from endoneurium. Based on his observations of the appearance of the cells under light microscopy, in 1910 Verocay ${ }^{36}$ argued that PNSTs arose from Schwann cells. The argument over the cell of origin for PNSTs continued through the 20th century. Masson ${ }^{18}$ argued that "the fundamental element of encapsulated neurinomas (schwannomas) is structurally identical with the syncytium of Schwann when that syncytium is undergoing autonomous proliferation." On the other hand, in his review of nerve sheath tumors, Wilder Penfield ${ }^{25}$ argued the opposite:

These tumors do not contain nerve fibers. The mistaken belief that they did do so led Verocay to call them neurinomas. They do not contain neuroglial elements, to the best of my belief after exhaustive study by means of special neuroglia methods. The type cell is not the Schwann cell but the connective tissue perineurial and endoneurial cell, as shown by the following facts. The tumor cells form fibroglia, collagen fibrils and elastic tissue. The outstanding histologic characteristic of these tumors is the presence in them of long parallel hairlike fibrils which stream together like hairs in a brush. They are not nerve fibrils. They are the architectural support without the nerve. They are reticulin fibrils staining with the same specific stains used for reticulin elsewhere...the Schwann cells, which seem to be analogous to neuroglia, could hardly be expected to form fibrils of this sort which bear no resemblance to neuroglia fibrils.

In 1940, Margaret Murray and Arthur Stout ${ }^{21}$ defined the arguments for and against Schwann cells as the origin of nerve sheath tumors:

Since the description by Verocay in 1908 of the specific nerve sheath tumor which he named neurinoma, two opposing views of its cellular origin have prevailed. One, represented by the work of Nageotte, and of Masson, is based on the study of the morphology and physiology of normal nerve sheaths under various experimental conditions. By comparing the histology of these tissues with that of the tumors, these workers feel that they have proved that the tumor originates from the sheath of Schwann. They therefore give it the name schwannoma, or peripheral glioma. The opposing opinion, represented by the work of Mallory and of Penfield, emphasizes mainly the study of the extracellular components of these tumors, and rests on the assumption that collagen and reticulin can be formed only by cells of mesodermal derivation. Since the tumors contain 
considerable collagen and reticulin, this line of reasoning induces the conclusion that the tumors are derived from the endoneural or perineural components of the nerve sheath, and the term perineural fibroblastoma is adopted.

Murray and Stout went on to culture normal nerve sheath and nerve sheath tumor cells in the laboratory. They found that the morphological characteristics of outgrowing cells was the same in both cultures and offered very compelling evidence that Schwann cells were the neoplastic cells in their tumor specimens. ${ }^{21}$

Further investigation into the histological characteristics of PNSTs revealed the presence of two different types of tumors: schwannomas and neurofibromas. As articulately described by Harkin and Reed ${ }^{1}$ in $1969,{ }^{1}$ a schwannoma "begins as a confined local growth that compresses the nerve around its periphery as it expands," while a neurofibroma is "composed of a tortuous plexus of Schwann cell cylinders and axons." Electron microscopy performed in the early 1990s confirmed the involvement of Schwann cells in the pathogenesis of schwannomas, yet there were several different cell types seen in neurofibromas, and identification of the actual tumor cell remained elusive. ${ }^{29}$

Advances in molecular and cellular biology have generated significant evidence indicating that Schwann cells are the cell of origin for both schwannomas and neurofibromas. ${ }^{13,20,26,28,29}$ Using immunohistochemical analysis, Stefansson and colleagues ${ }^{32}$ in 1982 reported that S100, a protein found normally only in Schwann cells, is also present in the predominant cell type of both plexiform and cutaneous neurofibromas. Furthermore, in 1990 Sheela et al. ${ }^{29}$ found that neurofibroma Schwann cells promote angiogenesis and are invasive in the chick chorioallantoic membrane model, yet neurofibroma fibroblasts neither promote angiogenesis nor are invasive.

The current model (reviewed by Gottfried et al. ${ }^{8}$ ) holds that in both schwannomas and neurofibromas the tumor cell is a Schwann cell completely lacking expression of the NF1 gene. The NF1 protein functions as a tumor suppressor by promoting the inactivation of the signal transduction protein Ras. Ras is activated when $G$ protein-coupled receptors replace GDP with GTP bound to Ras. Ras inactivation is caused by hydrolysis of GTP to GDP, an enzymatic process that is inherent in Ras but occurs at a slow rate. Neurofibromatosis Type 1 protein promotes the autohydrolysis of GTP to GDP in Ras, inactivating it. In schwannomas it is only the Schwann cell that is lacking the NF1 protein and, although it does secrete mitogens capable of functioning as both autocrine and paracrine growth factors, the supporting cells are otherwise normal. On the other hand, in patients with neurofibromatosis all of the supporting cells are haploinsufficient for NF1 and have a relative Ras hyperactivity, leading to increased proliferation in response to growth factors secreted by the tumorigenic Schwann cells.

A better understanding of the distinction between schwannomas and neurofibromas made a significant difference in their surgical management. Although schwannomas can usually be separated from the parent nerve and removed, it is often very difficult to do the same with neurofibromas. In the 1970s and 1980s, authors did not recommend attempted resection of neurofibromas, ${ }^{22}$ but advocated en bloc resection of the tumor and nerve with reconstruction. ${ }^{10,33}$ However, as described by Donner et al. ${ }^{5}$ in 1994, nonplexiform neurofibromas often only involve a few fasicles and with careful technique may be resected while sparing the parent nerve. Donner and associates ${ }^{5}$ reviewed their surgical experience in 263 patients with nerve sheath tumors and concluded:

With the exception of the rare plexiform tumors, benign nerve sheath tumors should be considered removable; however, neurofibromas have been believed by many to be unresectable due to a misleading histological appearance that suggests invasiveness. Our results indicate that neurofibromas as well as schwannomas can be removed, even from large nerve trunks, with an acceptable risk of injury to the nerve. Excision of solitary neurofibromas is possible, especially if an intrafascicular approach at both poles of the tumor and repeated NAP (nerve action potential) recording are used. Fascicles entering and leaving the tumor substance are usually nonfunctional, and their division usually does not lead to increased neurological deficit.

\section{Conclusions}

This brief review of surgery for peripheral nerve tumors illustrates how advances in the understanding of a disease have a profound impact on its surgical management. The ancient Greeks do not seem to have understood the function of nerves and so they did not describe peripheral nerve tumors, let alone develop any surgical intervention to treat them. In the 18th century, given the concern over lifethreatening inflammations after manipulation of a nerve, the accepted practice was either to remove the tumor en bloc with the parent nerve, or to amputate the affected limb. By the 19th century, with advances in nerve reapproximation, the goal of surgery was en bloc resection, followed by nerve anastomosis. In the 20th century, our understanding of the microscopic and structural differences between schwannomas and neurofibromas has made the practice of dissecting PNSTs from the parent nerve accepted. Today, further advances in our understanding of the molecular and genetic pathogenesis of PNSTs may hold even more rational approaches to the management of these tumors, perhaps even without surgery.

\section{References}

1. Cheselden W: The Anatomy of the Human Body, ed 6. London: William Bowyer, 1741

2. Cruickshank W: Experiments on the nerves, particularly on their reproduction; and on the spinal marrow of living animals. Phil Trans R Soc London 85:177-189, 1795

3. Cruveilhier J: Anatomie Pathologique de Corps Humain. Vol 1-2. Paris: Balliere, 1829-1842

4. Denmark A: An example of symptoms resembling tic douleureux produced by a wound in the radial nerve. Med-Chir Soc London 4:48-52, 1813

5. Donner TR, Voorhies RM, Kline DG: Neural sheath tumors of major nerves. J Neurosurg 81:362-373, 1994

6. Genersich A: Multiple neurome. Virchows Archiv 49:15-48, 1869

7. Goodrich JT: History of spine surgery in the ancient and medieval worlds. Neurosurg Focus 16(1):E2, 2004

8. Gottfried ON, Viskochil DH, Fults DW, Couldwell WT: Molecular, genetic, and cellular pathogenesis of neurofibromas and surgical implications. Neurosurgery 58:1-16, 2006

9. Haighton J: An experimental inquiry concerning the reproduction of nerves. Phil Trans R Soc London 85:190-201, 1795

10. Handler SD, Canalis RF, Jenkins HA, Weiss AJ: Management of brachial plexus tumors. Arch Otolaryngol 103:653-657, 1977

11. Harkin JC, Reed RJ: Tumors of the Peripheral Nervous 
System. Atlas of Tumor Pathology. Washington, DC: Armed Forces Institute of Pathology, 1969

12. Home E: An account of an uncommon tumor formed in one of the axillary nerves. Tr Soc Impr M Chir Knowl 2:152-163, 1800

13. Kamata Y: Study on the ultrastructure and acetylcholinesterase activity in von Recklinghausen's neurofibromatosis. Acta Pathol Jpn 28:393-410, 1978

14. Kennedy R: Nerve degeneration and regeneration. Phil Soc Glasgow 29:193-229, 1898

15. Krause F: Uber maligne Neurome und das Vorkommen von Nervenfasern in denselben. Leopzig: Breitkopf \& Hartel, 1887

16. Little KM, Zomorodi AR, Selznick LA, Friedman AH: An eclectic history of peripheral nerve surgery. Neurosurg Clin $\mathbf{N}$ Am 15:109-123, 2004

17. Lloyd GER (ed): Hippocratic Writings. New York: Penguin Books, 1978

18. Masson P: Experimental and spontaneous schwannomas (peripheral gliomas). Am J Pathol 8:367-416, 1932

19. McHenry LC Jr: Ancient origins, in Garrison FH (ed): History of Neurology. Springfield: Charles C. Thomas, 1969

20. McLaughlin ME, Jacks T: Thinking beyond the tumor cell: Nf1 haploinsufficiency in the tumor environment. Cancer Cell 1: 408-410, 2002

21. Murray MR, Stout AP: Schwann cell versus fibroblast as the origin of the specific nerve sheath tumor. Observations upon nerval nerve sheaths and neurilomomas in vitro. Am J Pathol 16: 41-60, 1940

22. Narakas A: Brachial plexus surgery. Orthop Clin North Am 12:303-323, 1981

23. Nutton V: Ancient Medicine. New York: Routledge, 2004

24. Odier L: Manuel de Medecine Pratique, ed 2. Paris: J. Paschoud, 1811

25. Penfield W: Tumors of the sheaths of the nervous system. Arch Neurol Psychiatry 27:1298-1309, 1932

26. Ratner N, Lieberman MA, Riccardi VM, Hong DM: Mitogen accumulation in von Recklinghausen neurofibromatosis. Ann Neurol 27:298-303, 1990

27. Sahlas DJ: Functional neuroanatomy in the pre-Hippocratic era: observations from the Iliad of Homer. Neurosurgery 48: 1352-1357, 2001

28. Sanguinetti C, Greco F, de Palma L, Specchia N, Toesca A, Nori
S: The ultrastructure of schwannoma and neurofibroma of the peripheral nerves. Ital J Orthop Traumatol 17:237-246, 1991

29. Sheela S, Riccardi VM, Ratner N: Angiogenic and invasive properties of neurofibroma Schwann cells. J Cell Biol 111:645-653, 1990

30. Smith RW: A Treatise on the Pathology, Diagnosis and Treatment of Neuroma. Dublin: Hodges and Smith, 1849

31. Souayah N, Greenstein JI: Insights into neurologic localization by Rhazes, a medieval Islamic physician. Neurology 65:125-128, 2005

32. Stefansson K, Wollmann R, Jerkovic M: S-100 protein in softtissue tumors derived from Schwann cells and melanocytes. Am J Pathol 106:261-268, 1982

33. Stevens JC, Davis DH, MacCarty CS: A 32-year experience with the surgical treatment of selected brachial plexus lesions with emphasis on its reconstruction. Surg Neurol 19:334-345, 1983

34. Swan J: A Dissertation on the Treatment of Morbid Local Affections of Nerves. London: J Drury, 1820

35. Syme J: Lectures on clinical surgery. Lecture XXII. Neuromata. Lancet 1:551-553, 1855

36. Verocay J: Zur Kenntnis der Neurofibroma. Beitr Pathol Anat 48:1-69, 1910

37. Virchow R: Die Krankhaften Geschwulste. Berlin: A Hirschwald, 1864

38. von Recklinghausen FD: Ueber die multiplen Fibrome der Haut und ihre Beziehung zu den multiplen Neuromen. Festschrift zur Feier des funfundzwanzigjahrigen Bestehens des pathologischen Instituts zu Berlin. Herrn Rudolf Virchow. Berlin: Hirschwald, 1882

39. Walker AE: A History of Neurological Surgery. Baltimore: Williams \& Wilkins, 1951

40. Wood W: Observation on neuroma. Trans Med-Chir Soc Edin 3:367-433, 1829

Manuscript submitted March 20, 2007.

Accepted April 13, 2007.

Address reprint requests to: Allan Friedman, M.D., Division of Neurosurgery, Duke University Medical Center, Box 3807 , Durham, North Carolina 27710. email: allan.friedman@ duke.edu. 